\title{
REPRESENTATION OF UNIVERSAL ALGEBRAS BY SHEAVES
}

\author{
U. MADDANA SWAMY
}

ABSTRACT. It is proved that every (universal) algebra $A$ with distributive and permutable structure lattice is isomorphic with the algebra of all global sections with compact supports of a sheaf of homomorphic images of $A$ over a topological space. This completely generalises the corresponding result of Klaus Keimel for l-rings.

Introduction. The following note is a preliminary report on the investigations undertaken by the author towards representing universal algebras by (continuous) sections of sheaves. The object of the present paper is to show that Keimel's general representation theorem [3, 3.3] for l-rings is valid for any universal algebra, with distributive and permutable structure lattice (see Theorem 2 below). The results in the general case are under investigation and will be communicated later.

In $\$ 1$ we define the (irreducible) spectrum of any (universal) algebra with distributive structure lattice and state its properties, whose proofs are routine. In $\$ 2$ we give a general construction of sheaves of algebras over a topological space, along the lines of Keimel [3] and apply this to get a general representation theorem for an algebra, with a distributive and permutable structure lattice. The crucial point of this note is Lemma 3, which replaces Lemma 1.11 of Keimel [3] where extensive use is made of the lring structure.

Throughout this paper, $A$ denotes an $\Omega$-algebra (algebra of type $\Omega$, see [2, p. 33]) and $\mathcal{L}(A)$ denotes its structure lattice. We refer to [4] for elementary basic properties of sheaves and [1] for preliminaries on sheaves of $\Omega$-algebras.

1. The spectrum of an $\Omega$-algebra. Let $\mathcal{L}(A)$ be distributive; as usual, we call a proper congruence $\phi$ on $A$ (meet) irreducible iff, for any $\theta_{1}, \theta_{2} \epsilon$ $\mathcal{L}(A), \theta_{1} \cap \theta_{2} \subseteq \phi$ implies either $\theta_{1} \subseteq \phi$ or $\theta_{2} \subseteq \phi$. The set $X$ of all irreducible congruences on $A$ becomes a topological space by taking $\left\{X_{\theta} \mid \theta \epsilon\right.$

Received by the editors May 30, 1973.

AMS (MOS) subject classifications (1970). Primary $18 \mathrm{~F} 20$.

Copyright $\odot 1974$, American Mathematical Society 
$\mathcal{L}(A)\}$ as open sets, where $X_{\theta}=\{\phi \in X \mid \theta \nsubseteq \phi\}$ for each $\theta \in \mathcal{L}(A)$, which we call the (irreducible) spectrum of $A$ and denoted by Spec $A$.

It is easy to see that the map $\theta \mapsto X_{\theta}$ is a lattice isomorphism of $\mathcal{L}(A)$ onto the lattice of all open subsets of $\operatorname{Spec} A$. A subset $Y$ of $\operatorname{Spec} A$ is closed iff every irreducible congruence which contains $\bigcap_{Y}\left(=\bigcap_{\phi \in Y} \phi\right)$ belongs to $Y$; and hence $Y$ is dense iff $\bigcap Y=\Delta$, the diagonal of $A \times A$. A subset $Y$ of $\operatorname{Spec} A$ is called full (following Keimel) iff every proper congruence on $A$ is contained in some member of $Y$.

2. General representation theorem. The following theorem and its proof are a nalogous to that of Keimel [3, Theorem 2.7].

Theorem 1. Let $X$ be a topological space and $A$, an $\Omega$-algebra. Let $\theta: X \rightarrow \mathfrak{L}(A)$ be a mapping such that for each $p \in A \times A, X(p)=\{x \in X \mid$ $p \in \theta(x)\}$ is open in $X$. Let $\mathcal{S}$ be the disjoint union of all $A / \theta(x), x \in X$, and define $\eta: S \rightarrow X$ by $\eta(s)=x$ for all $s \in A / \theta(x)$. For each $a \in A$, define $\hat{a}: X \rightarrow \mathcal{S}$ by $x \mapsto \pi_{x}(a)$, where $\pi_{x}: A \rightarrow A / \theta(x)$ is the natural epimorphism. Let $\mathfrak{S}$ be equipped with the largest topology that makes each $\hat{a}, a \in A$, continuous. Then we have the following:

(i) The family $\{\hat{a}(U) \mid a \in A, U$ is open in $X\}$ forms a base for the topology on $\mathcal{S}$.

(ii) The triple $\mathcal{F}=(\mathfrak{S}, \eta, X)$ is a global sheaf of $\Omega$-algebras over $X$.

(iii) The mapping $a \mapsto \hat{a}$ is a homomorphism of $A$ into $\Gamma(\mathcal{F})$, the $\Omega$-algebra of all global sections of the sheaf $\mathcal{F}$, and this is a monomorphism if and only if $\bigcap_{x \in X} \theta(x)=\Delta$.

(iv) $\mathfrak{F}$ is a $T_{2}$-sheaf if and only if $X$ is a $T_{2}$-space and $X(p)$ is closed also for all $p \in A \times A$.

If $\mathfrak{F}=(\mathfrak{S}, \eta, X)$ is any sheaf of $\Omega$-algebras, then for any two global sections $\sigma$ and $\tau$, we define, following Comer [1], $|(\sigma, \tau)|$ to be the set $\{x \in X \mid$ $\sigma(x) \neq \tau(x)\}$ and it is well known that $|(\sigma, \tau)|$ is always closed.

The following theorem now completely generalises the corresponding the orem of Keimel $[3,3.3]$ for l-rings.

Theorem 2. Let $A$ be an $\Omega$-algebra whose structure lattice is distributive and permutable and $Y$, a full and dense subspace of $X=\operatorname{Spec} A$. Let $Z$ be any topological space and $\zeta: Y \rightarrow Z$, a continuous map. Then there exists a sheaf $\mathcal{F}=(\mathfrak{S}, \eta, Z)$ of homomorphic images of $A$ over $Z$ such that $A$ is isomorphic with a subalgebra $\hat{A}$ of the algebra $\Gamma(\mathfrak{F})$ of all global sec- 
tions of $\mathcal{F}$, which contains all $\sigma \in \Gamma(\mathcal{F})$ such that $|(\sigma, \hat{a})|$ is compact for some $a \in A$, where $\hat{a}: Z \rightarrow \mathcal{S}$ is defined as in Theorem 1 .

We first prove a lemma which is crucial in proving the above theorem.

Lemma 3. Let $\mathcal{L}(A)$ be distributive and permutable and $Y$, a subspace of Spec $A$. For any $\theta \in \mathscr{L}(A)$, let $\theta^{*}=\bigcap_{\theta \nsubseteq \phi \in Y} \phi$. Let $\theta_{1}, \theta_{2}, \cdots, \theta_{n} \epsilon$ $\mathfrak{L}(A) ; a_{1}, a_{2}, \cdots, a_{n} \in A ; \bigcup_{i=1}^{n} \theta_{i}=A \times A$ and $\left(a_{i}, a_{j}\right) \in\left(\theta_{i} \cap \theta_{j}\right)^{*}$ for $1 \leq$ $i, j \leq n$. Then there exists $a \in A,\left(a, a_{i}\right) \in \theta_{i}^{*}$ for $1 \leq i \leq n$.

Proof. We observe that $\theta^{*}$ is the largest congruence on $A$ with the property $\theta \cap \theta^{*} \subseteq \bigcap Y$, and hence (i) $\theta \subseteq \phi$ implies $\phi^{*} \subseteq \theta^{*}$, and (ii) $\left(\theta \cap \phi^{*}\right) \cap \theta \subseteq \phi^{*}$ for all $\theta, \phi \in \mathscr{L}(A)$. Now, let $1 \leq i, j \leq n$ and $\theta=$ $\bigcap_{k=1}^{n}\left[\left(\theta_{i} \cap \theta_{k}\right)^{*} \cup\left(\theta_{k} \cap \theta_{j}\right)^{*}\right]$; then $\theta \subseteq\left(\theta_{i} \cap \theta_{k}\right)^{*} \cup\left(\theta_{k} \cap \theta_{j}\right)^{*}$ and therefore

$$
\theta \cap \theta_{k} \subseteq\left[\left(\theta_{i} \cap \theta_{k}\right)^{*} \cap \theta_{k}\right] \cup\left[\left(\theta_{k} \cap \theta_{j}\right)^{*} \cap \theta_{k}\right] \subseteq \theta_{i}^{*} \cup \theta_{j}^{*}
$$

for $1 \leq k \leq n$, which implies that

$$
\theta=\theta \cap\left(\bigcup_{k=1}^{n} \theta_{k}\right)=\bigcup_{k=1}^{n}\left(\theta \cap \theta_{k}\right) \subseteq \theta_{i}^{*} \cup \theta_{j}^{*}
$$

On the other hand, $\theta_{i}^{*} \cup \theta_{j}^{*} \subseteq\left(\theta_{i} \cap \theta_{k}\right)^{*} \cup\left(\theta_{k} \cap \theta_{j}\right)^{*}$ for $1 \leq k \leq n$ and hence $\theta_{i}^{*} \cup \theta_{j}^{*} \subseteq \theta$, so that, $\theta=\theta_{i}^{*} \cup \theta_{j}^{*}$

Therefore, we have $\left(a_{i}, a_{j}\right) \in \theta_{i}^{*} \cup \theta_{j}^{*}$ for $1 \leq i, j \leq n$ and now the lemma follows from the well-known Chinese remainder theorem (see [2, Problem 68, p. 221]). Q.E.D.

Proof of Theorem 2. For each open subset $U$ of $Z$, let $\theta_{U}=\bigcap \zeta^{-1}(U)$ and for each $z \in Z$, let $\theta_{z}=\bigcup\left\{\theta_{U} \mid U\right.$ is an open neighborhood of $\left.z\right\}$; observe that $\theta_{U}=\bigcap_{z \in U} \theta_{z}$ for each open subset $U$ of $Z$. For each $p \in A \times A$, $Z(p)=\left\{z \in Z \mid p \in \theta_{z}\right\}$ is open and hence by Theorem 1 , we have a sheaf $\mathcal{F}=(\mathfrak{S}, \eta, Z)$ of homomorphic images of $A$ over $Z$. Since $Y$ is dense, we have $\Delta=\bigcap Y=\theta_{Z}=\bigcap_{z \in Z} \theta_{z}$ and hence $a \mapsto \hat{a}$ is a monomorphism of $A$ onto the subalgebra $\hat{A}=\{\hat{a} \in \Gamma(\mathcal{F}) \mid a \in A\}$ of $\Gamma(\mathfrak{F})$.

Now, suppose $\sigma \in \Gamma(\mathcal{F}), a \in A$ and $|(\sigma, \hat{a})|$ is compact; then the usual argument shows the existence of open sets $U_{1}, \cdots, U_{n}$ of $Z$ and $a_{1}, a_{2}, \cdots$ $a_{n} \in A$ such that $\sigma / U_{i}=\hat{a}_{i} / U_{i}$ for $1 \leq i \leq n$ and $|(\sigma, \hat{a})| \subseteq \bigcup_{i=1}^{n} U_{i}$. Put $U_{0}=Z-|(\sigma, \hat{a})|$ and $a_{0}=a$; since $|(\sigma, \hat{a})|$ is closed and $\zeta$ is continuous, 
there exist $\theta_{i} \in \mathscr{L}(A)$ such that $\zeta^{-1}\left(U_{i}\right)=X_{\theta_{i}} \cap Y$ for $0 \leq i \leq n$. Also $\bigcup_{i=0}^{n} U_{i}=Z$ and therefore $Y \subseteq \bigcup_{i=0}^{n} X_{\theta_{i}}$, which implies that $\bigcup_{i=0}^{n} \theta_{i}=$ $A \times A$ (since $Y$ is full). We have $\hat{a}_{i} / U_{i} \cap U_{j}=\hat{a}_{j} / U_{i} \cap U_{j}$; that is $\left(a_{i}, a_{j}\right)$ $\in \bigcap_{z \in U_{i} \cap U_{j}} \theta_{z}=\theta_{U_{i} \cap U_{j}}=\bigcap \zeta^{-1}\left(U_{i} \cap U_{j}\right)$ and hence, following the notation of Lemma 3, we have $\left(a_{i}, a_{j}\right) \in\left(\theta_{i} \cap \theta_{j}\right)^{*}$ for $0 \leq i, j \leq n$. By Lemma 3, there exists $b \in A$ such that $\left(b, a_{i}\right) \in \theta_{i}^{*}$, for $0 \leq i \leq n$, and hence $\hat{b} / U_{i}=$ $\hat{a}_{i} / U_{i}=\sigma / U_{i}$, so that $\sigma=\hat{b}$. Q.E.D.

The class of algebras to which Theorem 2 applies is wide (see [5]) and includes $l$-rings, $l$-groups, regular rings, etc. Most of the results of Keimel [3] obviously hold for the class of algebras considered in this paper.

The author wishes to thank Professor N. V. Subrahmanyam for his valuable guidance in preparing this paper.

\section{REFERENCES}

1. S. D. Comer, Representation by algebras of sections over Boolean spaces, Pacific. J. Math. 38 (1971), 29-38. MR $46 \# 3412$.

2. G. Gratzer, Universal algebras, Van Nostrand, Princeton, N. J., 1968. MR 40 \#1320.

3. K. Keimel, The representation of lattice-ordered rings by sections in sheaves, Lecture Notes in Math., vol. 248, Springer-Verlag, Berlin and New York, 1971.

4. R. S. Pierce, Modules over commutative regular rings, Mem. Amer. Math. Soc. No. 70 (1967). MR $36 \# 151$.

5. A. F. Pixley, Clusters of algebras: Identities and structure lattices, Doctoral Dissertation, University of California, Berkeley, Calif., 1961.

DEPARTMENT OF MATHEMATICS, ANDHRA UNIVERSITY, WALTAIR 530003, INDIA 